\title{
Anomalous pulmonary venous drainage: a pictorial essay with a CT focus
}

\author{
Stephen Lyen ${ }^{1 *}$, Shalini Wijesuriya ${ }^{1}$, Eleanor Ngan-Soo ${ }^{1}$, Helen Mathias², Michael Yeong ${ }^{1}$, Mark Hamilton ${ }^{1}$ \\ and Nathan Manghat ${ }^{1}$
}

\begin{abstract}
The imaging of pulmonary venous anatomy has traditionally been performed with echocardiography and catheter pulmonary angiography. Magnetic resonance imaging (MRI) and notably multi-detector row computed tomography $(\mathrm{CT})$ have refined the usual imaging techniques.

Anomalous pulmonary venous drainage (APVD) is the drainage of one or more pulmonary veins outside the left atrium. Its detection is critical due to the strong association with congenital heart disease as well as other cardiac and respiratory anomalies, which have significant implications for patient management. The pervasive application of $C T$ combined with the relatively non-specific clinical presentation of APVD has resulted in the increased incidental detection of these anomalies. Knowledge is hence vital as the imaging specialist is now usually the first person to make such a diagnosis. Furthermore, pulmonary veins are an important site for arrhythmogenic foci and radiofrequency ablation of such sites is used in the treatment of refractory atrial fibrillation. Hence an imaging road map of these veins is crucial before any management can take place.

This pictorial review will illustrate embryology, normal and variant pulmonary vein anatomy, and varied patterns of APVD. Finally, we discuss the implications in the treatment of atrial fibrillation.
\end{abstract}

Keywords: Pulmonary veins, Drainage, Tomography X-ray computed, Magnetic resonance imaging

\section{Background}

There is a broad spectrum of pulmonary venous connections that deviate from the usual anatomical configuration and ranges from normal variant drainage to anomalous pulmonary-systemic communications, the latter arising from a failure of separation of the pulmonary and systemic circulations in-utero. Patients can be asymptomatic or present with non-specific cardiorespiratory symptoms. Diagnosis can be difficult, missed, or only made at late clinical presentation in adulthood.

Advances in non-invasive, cross-sectional imaging have refined radiological evaluation. Electrocardiogram (ECG)gated cardiac CT allows low dose, detailed anatomical assessment with excellent spatial and temporal resolution. Cardiac magnetic resonance imaging enables anatomical and physiological assessment, ventricular function and volumetrics and three-dimensional multiphase angiographic techniques. In addition, it enables quantification of shunt ratios (Qp:Qs). These techniques, including

\footnotetext{
* Correspondence: smlyen@doctors.org.uk

${ }^{1}$ Radiology Department, University Hospitals Bristol NHS Foundation Trust,

Bristol Royal Infirmary, Upper Maudlin St, Bristol BS2 8HW, UK

Full list of author information is available at the end of the article
}

echocardiography and chest radiography, must be regarded as complementary. The advantages and disadvantages of each technique in evaluating pulmonary venous connections are described in Table 1.

\section{Main text \\ Embryology of the pulmonary veins}

Understanding of the embryology is important in appreciating the wide patterns of APVD. Lung development commences at approximately 26 days of gestation with the respiratory diverticulum, arising from the primitive foregut [1]. The associated venous plexus (splanchnic plexus) communicates with the systemic cardinal and umbilicovitelline veins, thus forming the initial route of pulmonary venous drainage. At 27 to 29 days, an out-pouching develops from the postero-superior wall of the primitive left atrium [2]. This out-pouching subsequently begins to communicate with the pulmonary venous component of the splanchnic plexus. Simultaneously the systemic veins involutes, thus separating the two venous systems. In normal embryos there is complete dissociation of these venous systems which results in four separate pulmonary veins, which drain into the left atrium. Failure of this separation 
Table 1 Radiological assessment of the pulmonary veins

\begin{tabular}{|c|c|c|}
\hline & Advantages & Disadvantages \\
\hline Echocardiography & $\begin{array}{l}\text { - Non-invasive } \\
\text { - No ionising radiation } \\
\text { - Accessible } \\
\text { - Portable } \\
\text { - Real-time imaging } \\
\text { - Haemodynamic data }\end{array}$ & $\begin{array}{l}\text { - Limited evaluation of pulmonary veins and systemic } \\
\text { connections. } \\
\text { - Imperfect spatial resolution } \\
\text { - Operator dependent }\end{array}$ \\
\hline $\begin{array}{l}\text { Pulmonary } \\
\text { angiography }\end{array}$ & $\begin{array}{l}\text { - Haemodynamic data } \\
\text { - Pulmonary pressures } \\
\text { - Chamber oxygen } \\
\text { saturations }\end{array}$ & $\begin{array}{l}\text { - Invasive } \\
\text { - Limited information on associated congenital cardiothoracic } \\
\text { anomalies. } \\
\text { - Relationship to surrounding structures poorly defined. } \\
\text { - Radiation dose considerations } \\
\text { - Contrast allergy }\end{array}$ \\
\hline ECG-gated MDCT & $\begin{array}{l}\text { - Non-invasive } \\
\text { - Comprehensive thoracic anatomical assessment } \\
\text { - High spatial resolution } \\
\text { - Rapid image acquisition } \\
\text { - Can be used in patients with metallic implants } \\
\text { - Simultaneous coronary artery and cardiac morphological } \\
\text { evaluation } \\
\text { - Widely available } \\
\text { - No patient sedation. }\end{array}$ & $\begin{array}{l}\text { - lonizing radiation exposure } \\
\text { - Potential adverse iodinated contrast reaction } \\
\text { - Inferior temporal resolution to Echo and MRI } \\
\text { - Patient heart-rate control required }\end{array}$ \\
\hline Cardiac MRI & $\begin{array}{l}\text { - High temporal resolution } \\
\text { - No ionizing radiation } \\
\text { - Accurate ventricular functional analysis } \\
\text { - Accurate anatomical assessment } \\
\text { - Haemodynamic data } \\
\text { - 3D multiplanar reconstruction } \\
\text { - Quantification of shunt ratios. } \\
\text { - Non-contrast angiography is possible }\end{array}$ & $\begin{array}{l}\text { - Available in specialised centres } \\
\text { - Longer duration of image acquisition and analysis } \\
\text { - Images suboptimal with cardiac dysrhythmia or inability of patient } \\
\text { to breath hold } \\
\text { - Inferior spatial resolution to CT } \\
\text { - Patient claustrophobia } \\
\text { - Gadolinium contrast reactions. } \\
\text { - More prone to artefact. }\end{array}$ \\
\hline
\end{tabular}

process leads to the various types of anomalous venous drainage (Fig. 1). Failure of incorporation of the normal pulmonary veins into the left atrium may also occur (cor triatriatum), which will be discussed later.

\section{Normal pulmonary venous anatomy}

The usual arrangement consists of four separate pulmonary veins: right and left superior and inferior veins, which drain individually into the left atrium (Fig. 2). The right superior pulmonary vein (RSPV) drains the right upper and middle lobes of the lung with the right lower lobe drained by the right inferior pulmonary vein (RIPV). The left superior pulmonary vein (LSPV) drains the lingula and left upper lobe, whilst the left lower lobe is drained by the left inferior pulmonary vein (LIPV). This anatomical arrangement is found in $60-70 \%$ of the population [3].

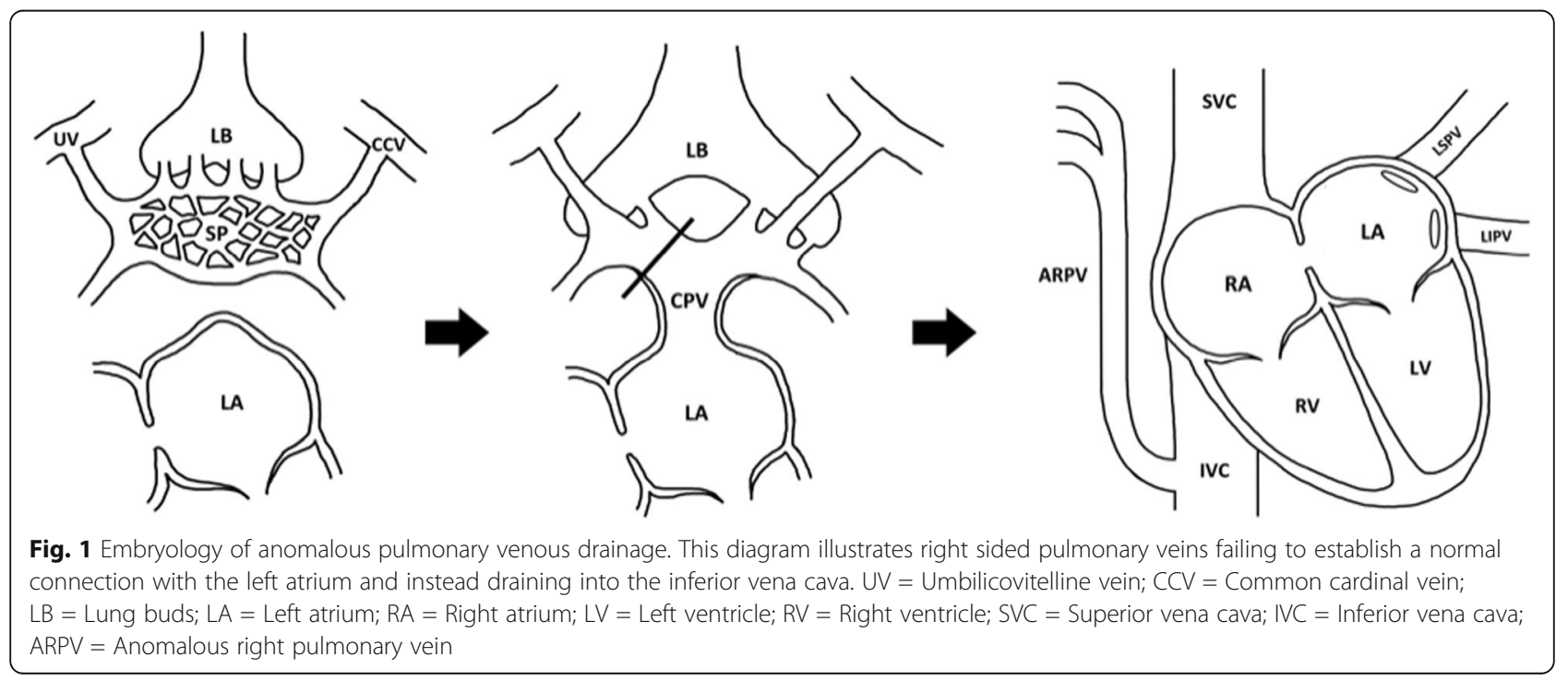




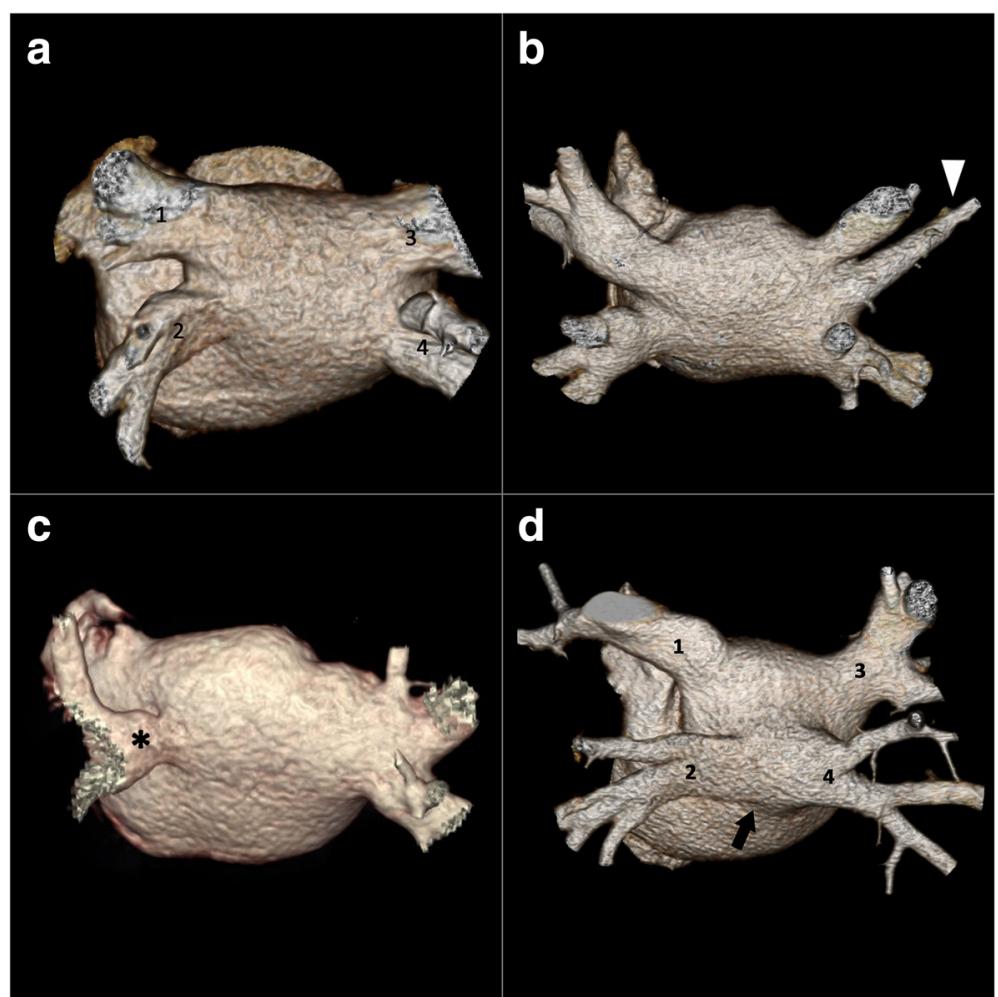

Fig. 2 Normal pulmonary venous anatomy and normal variants. 3D CT reconstructions of the left atrium and pulmonary veins, posterior views. a Normal pulmonary venous anatomy. (1) Left superior pulmonary vein, (2) Left lower pulmonary vein, (3) Right superior pulmonary vein, (4) Right inferior pulmonary vein. b Normal variant right middle pulmonary vein (arrowhead). c Single conjoined left pulmonary vein (asterisk). d Anatomical variant with conjoined ostium (black arrow) of the left inferior pulmonary vein (2) and right inferior pulmonary vein (4)

\section{Normal variant pulmonary venous drainage}

A wide variation of normal variant pulmonary venous drainage exists and is a common incidental finding. Normal variants typically consist of conjoined or accessory veins [4]. A conjoined (or common) vein is present when the superior and inferior veins on the same side unite to form a single confluence before entering the left atrium, resulting in a single atriopulmonary venous junction (or ostium). They are more common on the left (Fig. 2). Rarely this can occur via a common superior or inferior conjoined vein and can look similar to repaired total anomalous pulmonary venous drainage (TAPVD) on imaging (Fig. 2).

Accessory (or supernumerary) veins (Fig. 2) are separate from the superior or inferior pulmonary veins with an independent drainage (and atriopulmonary venous junction) into the left atrium. In contrast to a conjoined vein, these supernumerary variants usually have a narrower ostium than normal [5]. Accessory pulmonary veins may be seen in $30 \%$ of patients [6]. In a study examining left atrial anatomy in patients with and without atrial fibrillation (AF) who had CT angiography, there was no significant difference in number of pulmonary veins, although the left atria and pulmonary ostial dimensions were larger in AF patients [7]. Accessory veins are more common on the right, of which the most frequent variant is an accessory vein draining the middle lobe, seen in up to $26 \%$ of patients [8].

\section{Anomalous pulmonary venous drainage}

Partial anomalous pulmonary venous drainage (PAPVD) describes the connection of at least one pulmonary vein, but not all, to the systemic venous system or right atrium (RA). Drainage of all pulmonary veins outside the left atrium is termed total anomalous pulmonary venous drainage (TAPVD).

\section{A. Partial anomalous pulmonary venous drainage (PAPVD)}

The prevalence of PAPVD has been reported to be between $0.4-0.7 \%$ [9]. It is more common on the right and results in the formation of a left-to-right shunt.

Right-sided anomalous drainage may occur into any of the central veins. The most common form of PAPVD is the anomalous drainage of the RSPV into the superior vena cava (SVC) [10]. This frequently occurs at the junction of the SVC and RA (Fig. 3) [11]. Occasionally, drainage occurs at a higher level at the junction between 


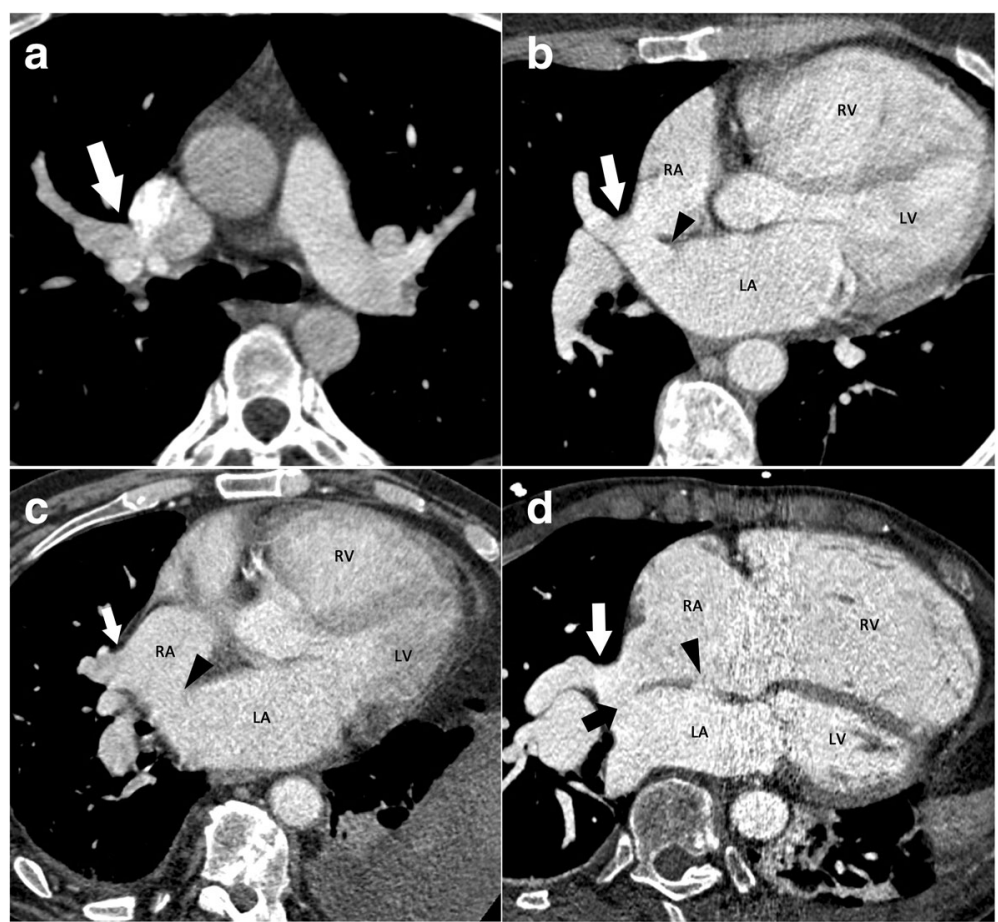

Fig. 3 Different variations of anomalous right superior pulmonary vein. (a) Axial image at the level of the main pulmonary artery. Right superior pulmonary vein (RSPV) draining into the superior vena cava (SVC) only (arrow). No sinus venosus defect was demonstrated. (b) Axial oblique CT. RSPV with a connection to the superior vena cava/right atrium (SVC/RA) confluence (arrow), but then continuing to drain into the left atrium (arrowhead). No direct sinus venosus defect. The right ventricle is dilated. (c) Axial oblique CT. RSPV draining into the SVC/RA confluence (white arrow) with a superior sinus venosus defect (black arrowhead) and right ventricular dilatation. (d) Axial oblique CT image. Anomalous RSPV is seen draining into the SVC (white arrow). Superior sinus venosus defect is demonstrated (black arrow) as well as an atrial secondum defect (black arrowhead)

the SVC and brachiocephalic vein above the level of the azygos vein, or into the azygos vein itself (Fig. 4). Other right-sided pulmonary-systemic connections include drainage into the coronary sinus, inferior vena cava (IVC) or drainage of all right pulmonary veins into the RA. Anomalous pulmonary venous of the right superior pulmonary veins is commonly associated with superior sinus venosus atrial septal defects, an atrial septal defect being the most common associated congenital heart defect (Figs. 3, 4 and 5) [12]. The identification of these abnormalities has a significant bearing on surgical management as anomalous pulmonary veins could be rerouted to the left atrium via an atrial septal defect [13].

Up to $18.2 \%$ of PAPVD cases affect the left-sided pulmonary veins [12]. The commonest pattern on the left is the LSPV connected to the left brachiocephalic vein (Figs. 6 and 7) [10]. In this condition, a vertically orientated vessel courses lateral to the aortic arch prior to draining into the left brachiocephalic vein. This anomalous vein can sometimes be misdiagnosed as a left-sided SVC on CT (Fig. 7) [10, 14]. The two anomalies can be differentiated at the level of the left hilum. Under normal conditions a single vessel is seen anterior to the left main bronchus, the LSPV. In PAPVD this vessel is absent, whereas in persistent left SVC two vessels lie anterior to the left main bronchus, the anomalous left superior pulmonary vein and the persistent left SVC. The course of the lobar intraparenchymal veins may also be appreciated as they drain into the anomalous vessel. A further feature of left upper lobe PAPVD is a normal or enlarged left brachiocephalic vein, whereas in a persistent left SVC the left brachiocephalic vein may be absent or small.

Other described left-sided connections include drainage into the hemiazygos vein or the coronary sinus [15].

Careful scrutiny of the pulmonary veins is important and the identification of less than four pulmonary veins connected to the left atrium should alert the reader to a diagnosis of PAPVD. However, ancillary features may be present which may also allude to this diagnosis. In the presence of a left-to-right pulmonary shunt, enlargement of the right heart may occur due to increased volume loading (Figs. 3, 4 and 5), dependent on the presence of an intact atrial septum. In addition, the vessel receiving the anomalous vein may be enlarged and there may be asymmetry of the pulmonary veins. The magnitude of the left-to-right shunt is dependent of the number 

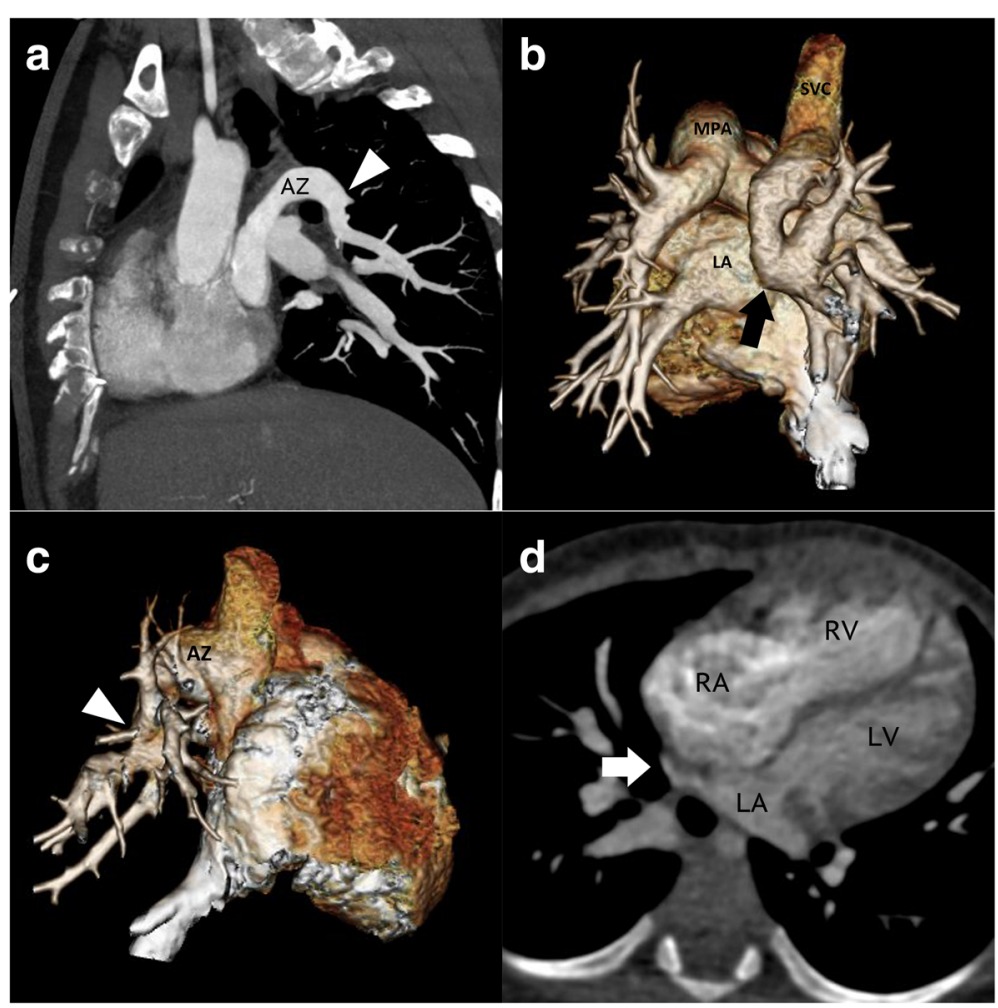

Fig. 4 Anomalous drainage into the azygos vein. Images $a$ and $b$ are from an adolescent patient with anomalous pulmonary venous drainage into the azygos vein. Images $\mathrm{c}$ and $\mathrm{d}$ are from a paediatric patient with anomalous pulmonary venous drainage identified on echocardiography. a Sagittal maximum intensity projection (MIP) CT image showing the right superior pulmonary vein (white arrow) draining into a dilated azygos vein (labelled AZ). $\mathbf{b}$ Three-dimensional (3D) CT reconstruction of patient in (a), posterior view. The black arrow indicates the right superior pulmonary vein draining into the azygos vein. c 3D CT reconstruction. Most of the right sided pulmonary veins are seen draining into a dilated azygos vein (labelled AZ). $\mathbf{d}$ Axial $C T$ image of the patient described in (c). Only a very small right middle pulmonary vein is seen draining normally into the left atrium (white arrow). Note the dilated right atrium and right ventricle consistent with a significant left-to-right shunt

PAPVD. In a series looking at single PAPVD, the shunt is modest (Qp:Qs $\sim 1.3-1.6)$ associated with mild dilatation of the right ventricle [16]. On top of volume loading to the right ventricle, APVD is one of the treatable causes of pulmonary hypertension in adults [17].
Congenital cardiac and thoracic anomalies, for example thoracic hypoplasia and cardiac dextro/levoversion may also occur, the presence of which should prompt examination of the pulmonary venous anatomy (Figs. 8, 9 and 10).

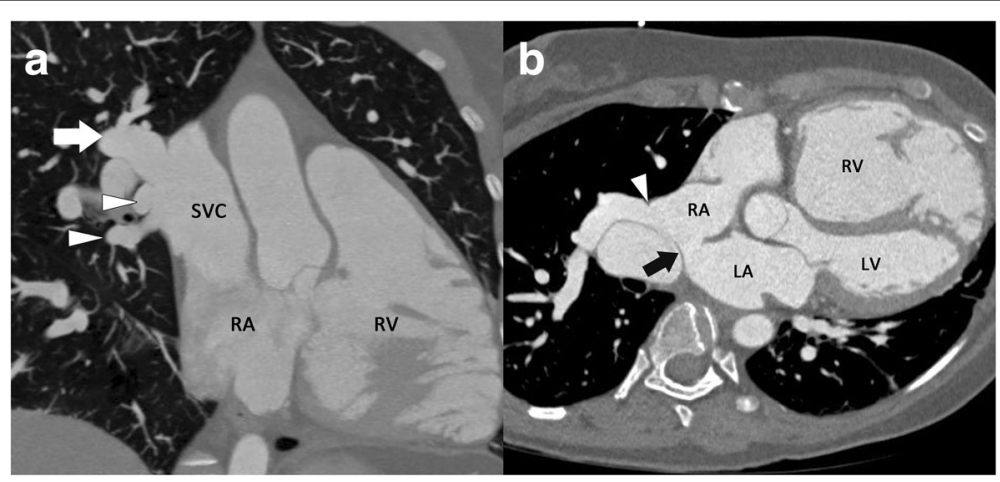

Fig. 5 Anomalous right superior and middle pulmonary veins. (a) Coronal oblique maximum intensity projection CT image. The right superior pulmonary vein is seen draining into the superior vena cava (arrow). Just inferior to this the right superior and inferior middle pulmonary veins are also seen draining separately into the superior vena cava/right atrium confluence (arrowheads). b Axial oblique MIP CT 5-chamber view, demonstrating a superior sinus venosus defect (black arrow), anomalous middle pulmonary vein (white arrowhead) and marked right ventricular dilatation secondary to the left-to-right shunt 


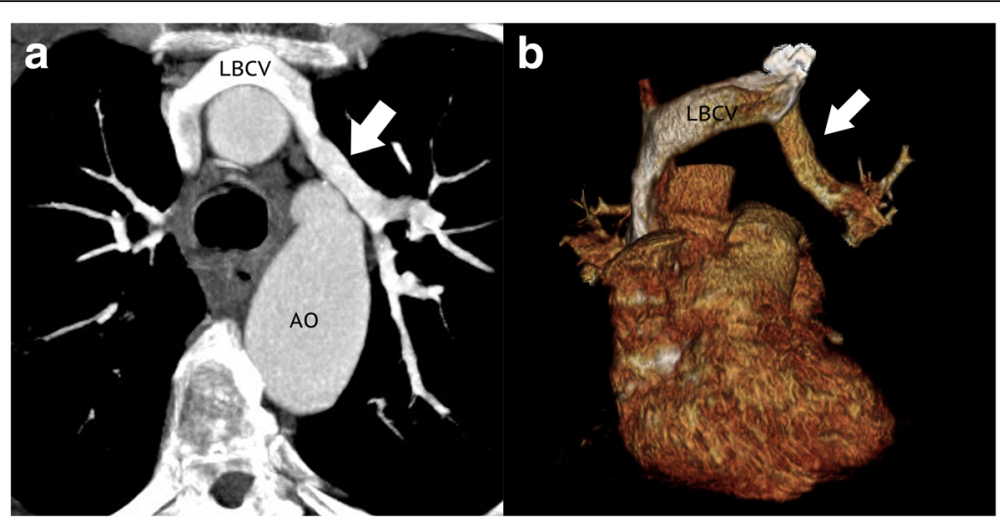

Fig. 6 Anomalous left superior pulmonary vein. a Axial oblique maximum intensity projection (MIP) $\subset$ T image. Anomalous left superior pulmonary vein (LSPV) drains into the left brachiocephalic vein (LBCV) (white arrow). AO - Aorta. b Three-dimensional CT reconstruction demonstrating the anomalous LSPV draining into the LBCV (white arrow)

Cross-sectional imaging with CT and MRI is widely used for the diagnosis of APVD in the adult population. CT has superior spatial resolution and thus is useful in presurgical planning in complex lesions. However, MRI is often sufficient for diagnosis and has the advantages of providing functional assessment and shunt calculation.
In addition, MRI does not require ionising radiation which is of particular importance in young patients.

\section{Scimitar (hypogenetic lung/venolobar) syndrome}

Scimitar syndrome consists of right-sided anomalous pulmonary venous drainage, hypoplasia of the lung and

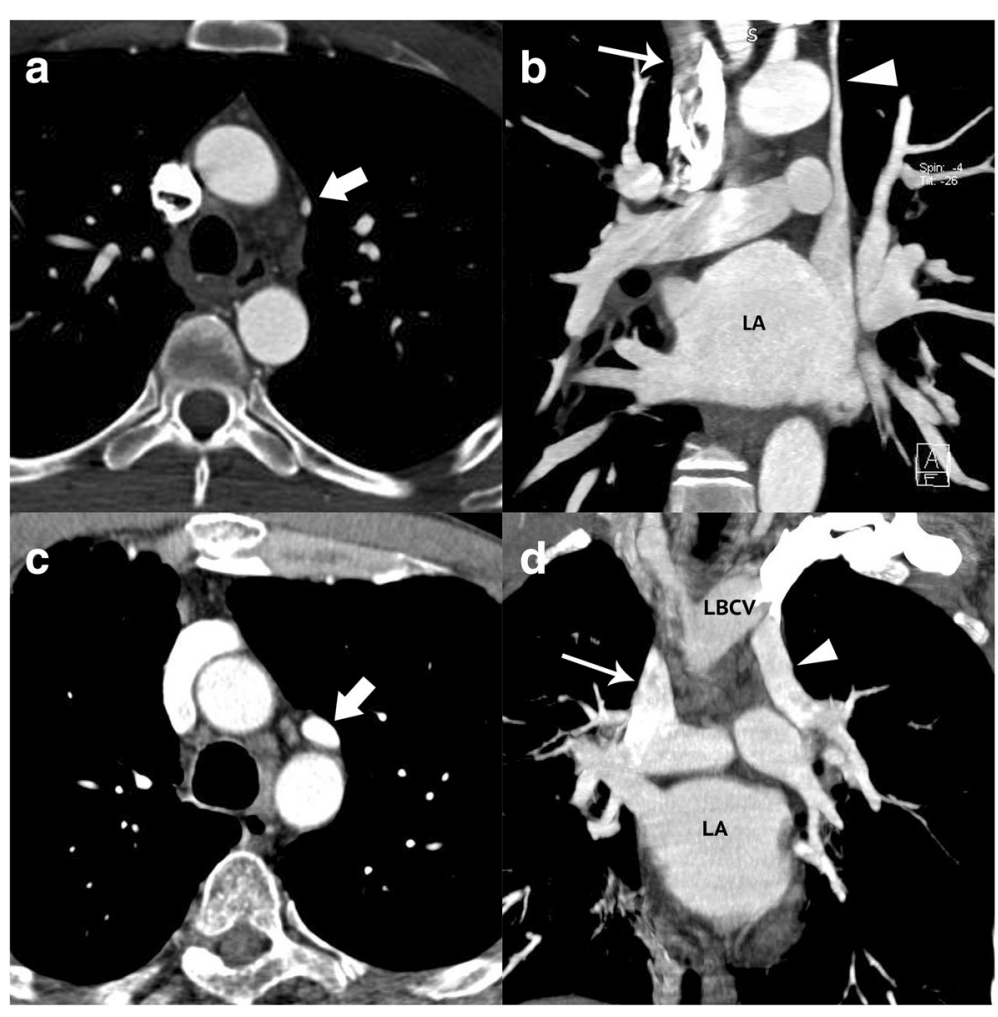

Fig. 7 Anomalous left superior pulmonary vein (LSPV) may mimic a left sided superior vena cava (SVC) on axial CT images. Patient 1. Bilateral SVCS with anomalous left SVC draining into the left atrium (a and b). a Bilateral SVCs. Right SVC (arrowhead) and small left sided SVC (white arrow). b Coronal MIP CT in the same patient showing the anomalous left SVC draining into the left atrium (white arrow). Normal right-sided SVC (thin white arrow). Patient 2. Partial anomalous pulmonary venous drainage (c and d). c Anomalous LSPV (white arrow), which on single axial slice is similar in appearance to the left-sided SVC in (a). d Coronal MIP CT showing the anomalous LSPV (white arrow) draining into the left brachiocephalic vein (LBCV). Normal right SVC (thin white arrow) 


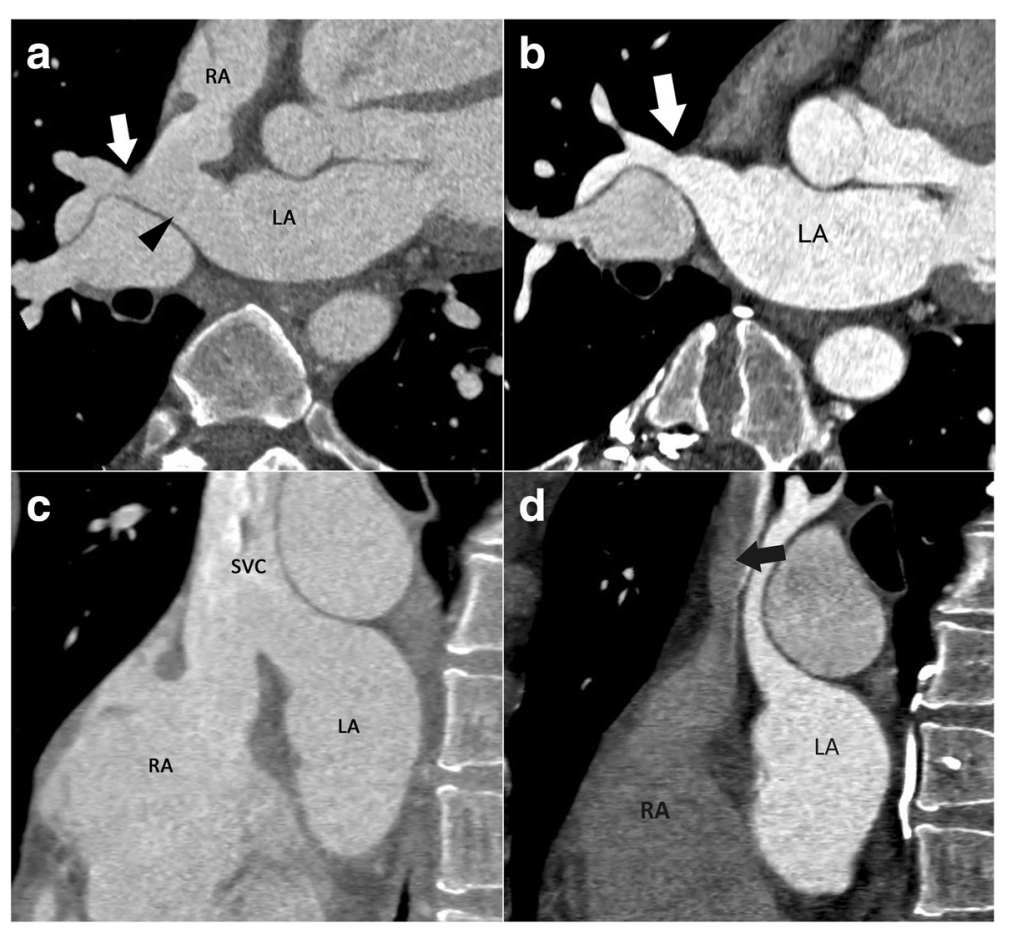

Fig. 8 Anomalous right-sided pulmonary venous drainage with a superior sinus venosus defect. a Axial oblique CT MPR images show an anomalous right superior pulmonary vein (RSPV) draining into the superior vena cava/right atrium (SVC/RA) junction (white arrow) with an associated superior sinus venosus defect (SSVD, arrowhead) connecting the left atrium (LA) and right atrium (RA). b Post surgical repair the right superior pulmonary vein is now directed into the left atrium with closure of the SSVD. c Sagittal oblique CT images showing the SVC overriding a SSVD between the two atria. d Post surgery the SSVD has been repaired, the SVC (black arrow) drains directly into the right atrium

pulmonary artery, cardiac dextroposition (Fig. 9) and systemic arterial supply to the right lower lobe. The latter may occur either from the descending thoracic or upper abdominal aorta [18]. The anomalous right-sided drainage occurs most commonly into the subdiaphragmatic IVC (Fig. 9) [19]. Drainage may also occur into the porto-hepatic veins, azygos vein, coronary sinus, right atrium or the suprahepatic portion of the IVC.

Scimitar syndrome can also be associated with pulmonary arteriovenous malformations, abnormalities of the diaphragm and genitourinary tract. Whilst usually right-sided, left-sided cases have been described in the literature [20].

The scimitar is a curved traditional sword held by Persian and Turkish warriors, from which the syndrome receives its name. The imaging findings on chest $x$-ray are classic. The anomalous vein is seen as a curved, tubular structure noted lateral to the right heart border, coursing inferiorly (Scimitar sign) (Fig. 9).

A pseudoscimitar syndrome occurs when the above constellation of findings is associated with an anomalous vein that takes a tortuous course and drains into the left atrium, rather than the IVC, producing a false positive Scimitar sign on chest radiography. This has been coined the pseudoscimitar vein, also known as a meandering right pulmonary vein $[10,21]$.

\section{PAPVD: Chest radiography}

Patients with PAPVD often have non-specific cardiac or respiratory symptoms and are initially investigated with chest radiography, which can be completely normal. However, PAPVD should be suspected with the following findings:

- An usual course of a central venous catheter [22].

- Focal mediastinal widening to the left of the aortic knob (Fig. 11).

This may be caused by an anomalous left superior pulmonary vein.

- Scimitar vein and other associated findings such as right lung hypoplasia and cardiac dextroposition.

- Unusual patterns of pulmonary oedema.

Left upper lobe PAPVD will spare vascular congestion of the left upper lobe in left heart failure, as the left upper 


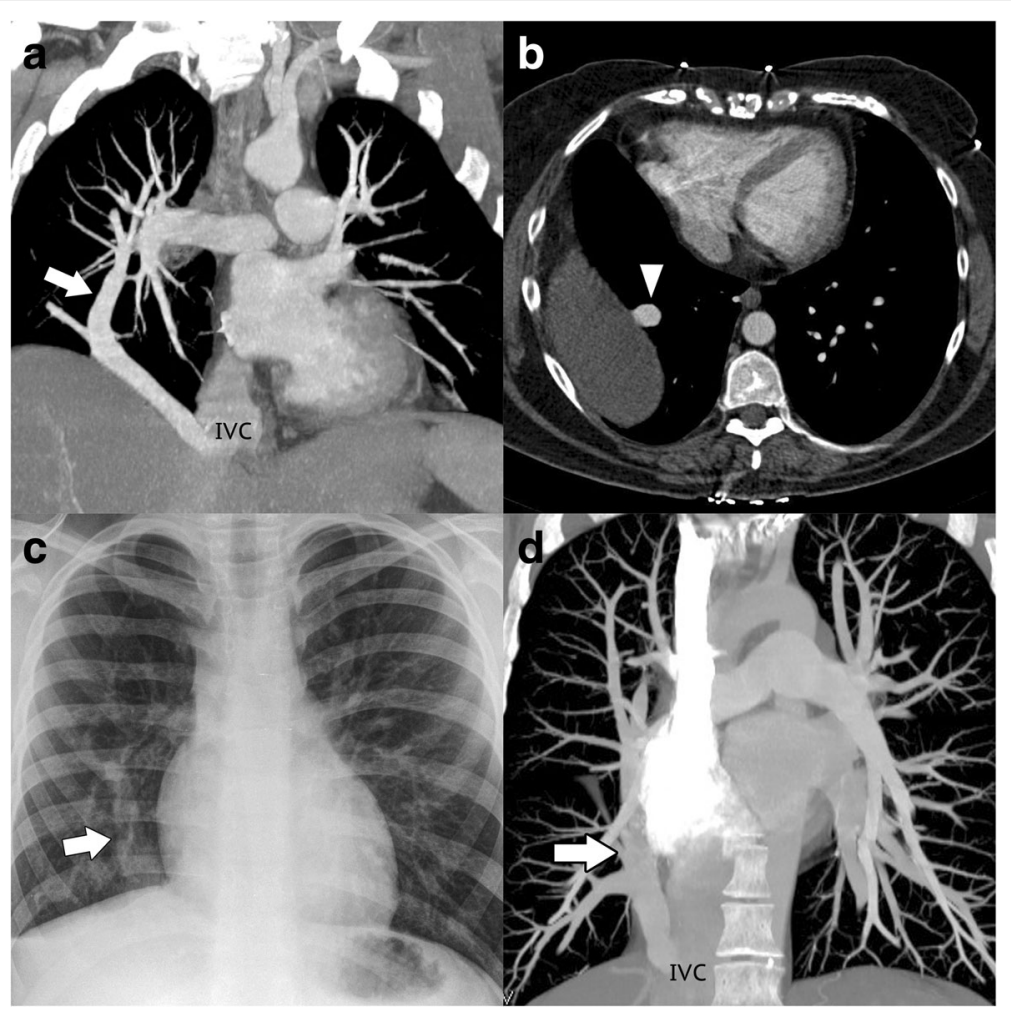

Fig. 9 Scimitar Syndrome. a Coronal oblique maximum intensity projection (MIP) CT images. A large right sided pulmonary vein is seen supradiaphragmatic inferior vena cava (IVC) in a patient with Scimitar Syndrome. b Axial CT image of same patient as in (a). The white arrow indicates the anomalous right pulmonary vein. The asymmetry of the pulmonary vasculature provides a clue to identifying the abnormality. The right hemidiaphragm is elevated consistent with hypoplasia of the right lower lobe, a common association with anomalous pulmonary venous drainage. c Chest radiograph of a paediatric patient, demonstrating a tubular opacity in the right paracardiac region consistent with a scimitar vein (arrow). d Coronal MIP CT image showing a large right pulmonary vein draining into the infradiaphragmatic IVC

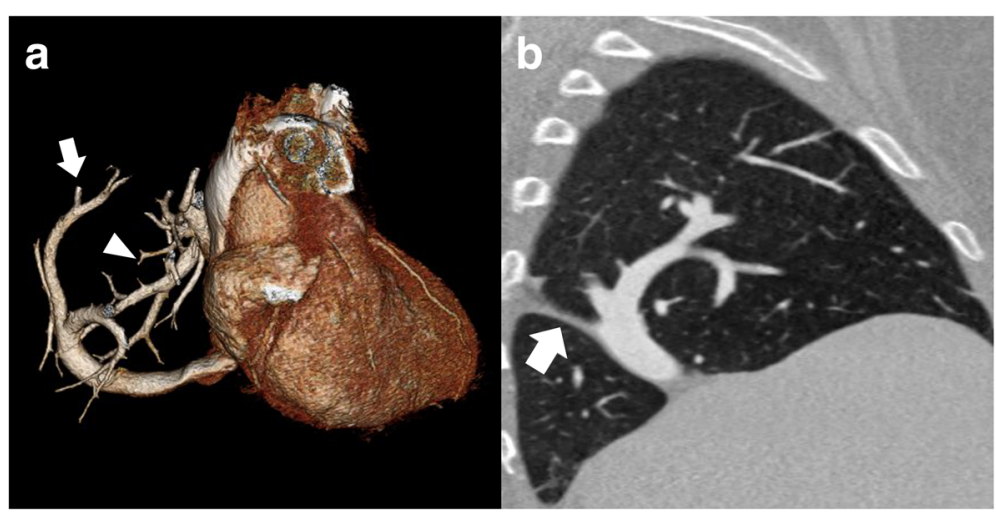

Fig. 10 Anomalous right inferior pulmonary vein drain. Adult patient presenting with breathlessness. a Three-dimensional (3D) CT reconstruction. A large right inferior pulmonary vein is seen draining most of the right upper lobe into the supradiaphragmatic inferior vena cava (arrow). Most of the lower lobe is seen draining normally into the left atrium (arrowhead). b Sagittal MIP CT image shows a hypoplastic right lower lobe (arrow - oblique fissure). The large curvilinear right pulmonary vein is seen draining the right upper lobe 


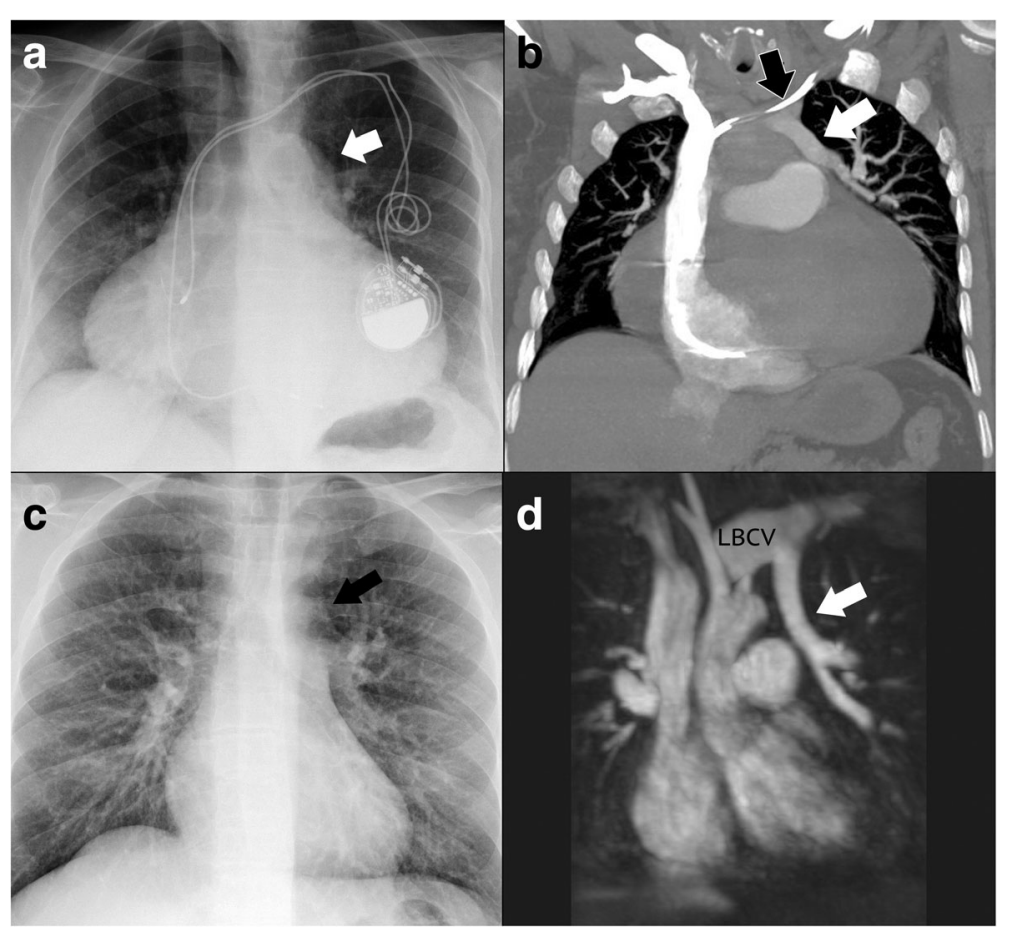

Fig. 11 Chest radiograph findings in anomalous pulmonary venous drainage- Focal mediastinal widening to the left of the aortic knob. a Patient 1. Postero-anterior (PA) chest radiograph (CXR). Vascular shadow adjacent to the aortic knuckle corresponds to anomalous left superior pulmonary vein (LSPV) in (b). b Coronal maximum intensity projections (MIP) CT image demonstrating anomalous LSPV (black arrow) draining into the brachiocephalic vein (LBCV). This is the most common left sided partial anomalous pulmonary venous drainage pattern. c Patient 2. Posteroanterior chest radiography. The LSPV can be seen as a prominent vascular shadow lateral to the aortic knuckle and left subclavian artery (black arrow). d Coronal MIP MR angiography demonstrating LSPV (arrow) draining into the left BCV (LBCV)

lobe pulmonary vein does not drain into the left atrium. Similarly, in right heart failure the same anomaly causes pulmonary oedema isolated to the left upper lobe [23].

- Effects of longstanding left-to-right shunts (Fig. 12).

These include enlargement of the cardiac silhouette (in particular right ventricular dilatation), pulmonary plethora, right hilar vascular prominence and dilatation of the central pulmonary arteries.

The above findings are summarised in Table 2.

\section{Cor Triatriatum}

This rare condition refers to failure of incorporation of normal pulmonary veins into the left atrium [9] and accounts for up to $0.1 \%$ of congenital heart disease [24]. There is separation of the left atrium into two chambers, separated by a thin, fenestrated membrane. The posterior chamber drains the pulmonary veins and the anterior chamber (true left atrium) drains into the left ventricle via the mitral valve, overall resulting in an obstruction to the drainage of the pulmonary veins. Patients can present with failure to thrive and congestive heart failure.

\section{B. Total anomalous pulmonary venous drainage (TAPVD)}

This refers to drainage of all four of the pulmonary veins to another cardiovascular structure other than the left atrium [25] and accounts for about $2 \%$ of cardiac malformations. Four types exist, depending on the level of drainage:

Type 1 - supracardiac: This accounts for up to $55 \%$ of cases and is the most common. It typically involves convergence of all four pulmonary veins behind the heart to form a common vein (Fig. 13). This then drains into the left brachiocephalic vein and henceforth into the SVC. This can cause the classic 'snowman' or 'figure-of eight appearance' on chest radiography.

Type 2 - abnormal communication at the cardiac level: drainage occurs into the RA or coronary sinus.

Type 3 - drainage below the level of the heart or diaphragm, for example into the IVC, portal or hepatic veins. 


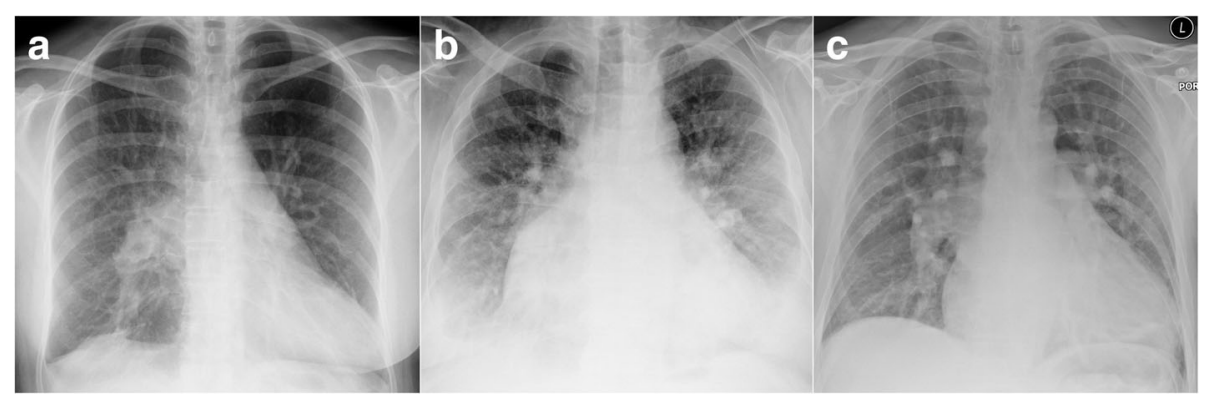

Fig. 12 Effects of a longstanding left-to-right shunt in patients with PAPVD. a Right superior pulmonary vein (RSPV) draining into the superior vena cava (SVC). Right sided pulmonary plethora is seen. b Right atrial dilatation, cardiomegaly and features of pulmonary oedema. Subsequent computed tomographic pulmonary angiography (CTPA) demonstrated a RSPV draining into the SVC with dilated right heart chambers. c Patient with anomalous right superior and middle pulmonary veins draining into the superior vena cava/right atrium confluence. A chest radiograph demonstrates cardiomegaly and central pulmonary artery dilatation secondary to long-term left-to-right shunt

Type 4 - drainage at more than one level (Fig. 13).

In patients with TAPVD, a right-to-left shunt is crucial for early survival and this manifests usually in the form of a patent foramen ovale or an atrial septal defect. Patients present early in the neonatal period with symptoms of congestive cardiac failure and cyanosis. Prompt surgical repair is essential, thus it is rarely encountered on CT or MRI. In the vast majority of cases, echocardiography is the mainstay of diagnosis and anatomical description of TAPVD. Cross-sectional imaging is usually reserved for when the diagnosis is unclear, or incomplete definition of the pulmonary veins at echocardiography [26].

TAPVD is commonly associated with other congenital cardiac lesions. A large international multicentre study conducted by Seale et al. $(n=422)$ showed $14 \%$ of patients had associated cardiac lesions, and that if definitive repair was not performed at the time of surgery this was an independent risk factor for death [27]. In addition, pre- and postoperative pulmonary vein obstruction or stenosis were also independent risk factors for death in multivariate analysis. This highlights the

Table 2 Features of PAPVD on chest radiography

\footnotetext{
- May appear normal

- Unusual course of a central venous catheter

- Focal widening of the mediastinum to the left of the aortic knob

- Unusual patterns of pulmonary oedema

- The Scimitar vein

- Effects of a longstanding shunt - dilatation of the right heart chambers and central pulmonary arteries.

- Cardiac and thoracic associations eg: pulmonary hypoplasia and cardiac dextroposition in Scimitar syndrome.
}

importance of accurate pre- and postoperative anatomical evaluation in this cohort of patients.

\section{Relationship between pulmonary veins and atrial fibrillation}

Atrial fibrillation (AF) is the most common cardiac arrhythmia, affecting $5 \%$ of the population of the United Kingdom over the age of 65 years, increasing to $10 \%$ above the age of 75 years [28]. Patients with $\mathrm{AF}$ are at a greater risk of stroke, increasing from $1.5 \%$ from ages $50-59$ years to $23.5 \%$ in those aged 80-89 years [29]. Mortality rates are also higher in these patients [30].

One of the mechanisms believed to trigger the onset of AF is the production of ectopic atrial beats [4]. The pulmonary veins have been found to be an important site of such arrhythmogenic activity, with up to $96 \%$ of foci triggering paroxysmal episodes of AF arising from them [31]. This may be explained by the observation of sleeves of myocardial tissue from the left atrium extending into the pulmonary veins, with distances varying from 2 to $17 \mathrm{~mm}$ [32]. Ectopic foci most commonly arise from the left vssuperior pulmonary vein. This may be explained by two observations: myocardial sleeves are longer in the superior pulmonary veins and thicker at the atriopulmonary venous junction [33]. Radiofrequency ablation (RFA) has been successfully used to disconnect the electrical connection between the pulmonary veins and the left atrium, thereby treating AF in affected patients [34].

\section{The role of $\mathrm{CT}$ in the mapping of pulmonary vein} anatomy - what the electrophysiologist needs to know Given the variability of pulmonary venous anatomy, detailed pre-procedural evaluation of the left atrium 


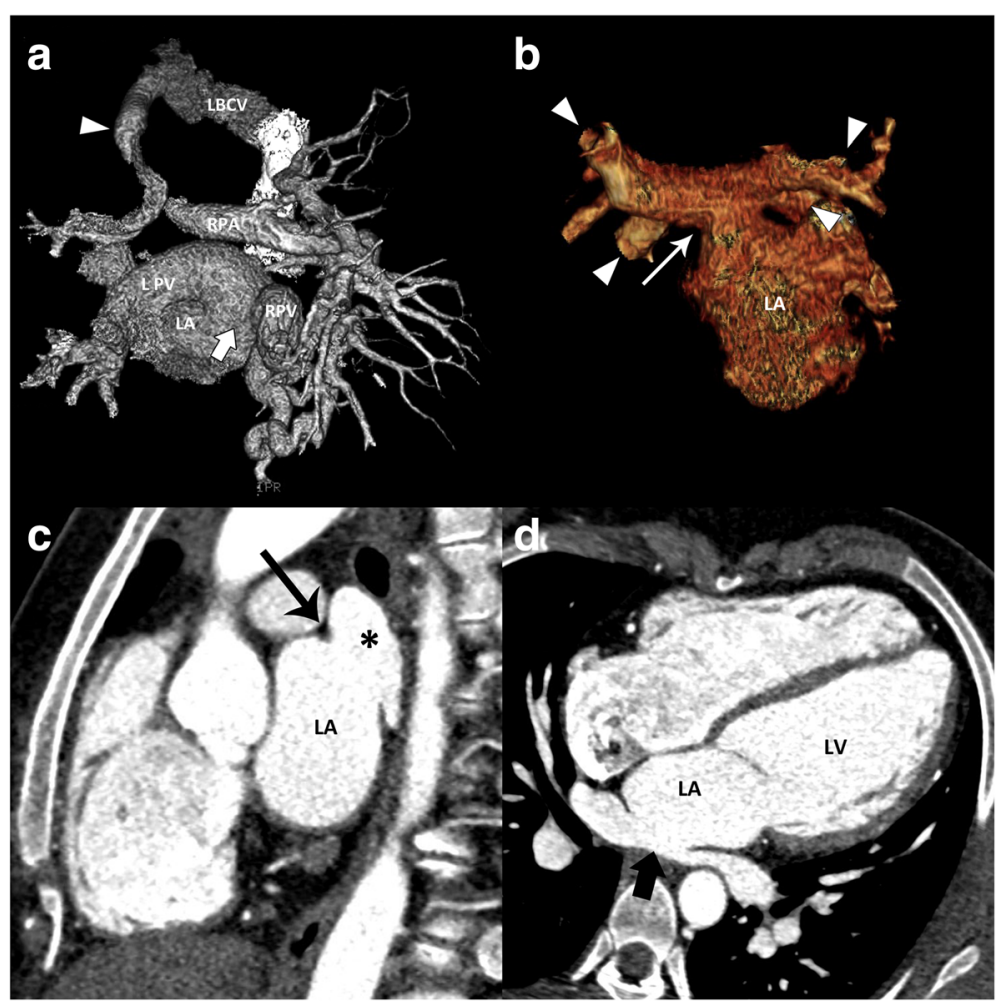

Fig. 13 Total anomalous pulmonary venous drainage (TAPVD) repair. a Three-dimensional (3D) CT reconstruction. An adult patient presenting with breathlessness had a computed tomographic pulmonary angiogram (CTPA). A single left pulmonary vein (LPV) crosses the midline to form a single confluence with the right pulmonary veins (RPV) and joins the left atrium (LA) via a baffle (white arrow). A residual anomalous left superior pulmonary vein (white arrowhead) is seen draining into the left brachiocephalic vein (LBCV); presumed previous type 4 TAPVD. Incidentally the pulmonary vessels in the right lower lobe were tortuous and dilated with a suspected arteriovenous malformation. b Three dimensional (3D) CT reconstruction. A paediatric patient with a Type 2 TAPVD repair. The pulmonary veins (white arrowheads) can be seen forming a single venous confluence posterior to the LA. This has been reconnected to the LA via a small baffle (white arrow). c Sagittal CT image. Type 2 TAPVD. A single venous confluence of the pulmonary veins is present (asterisk) posterior to the left atrium (LA) with a focal narrowing where this has been surgically connected to the LA (black arrow). d Axial CT image. This again shows the posterior venous confluence of the pulmonary veins (black arrow)

and the pulmonary veins is desirable prior to treatment procedures like RFA. This may be an arduous task during angiography and may only serve to increase procedure times (and consequently radiation doses) [35]. Therefore, pre-procedural CT plays an integral role in the diagnostic and treatment algorithms.

As mentioned earlier, supernumerary veins have smaller ostia than normal. Subsequently, they may be difficult to identify during fluoroscopic evaluation and any ectopic foci arising from them may go untreated, increasing the likelihood of recurrence [36].

RFA is performed at or within $5 \mathrm{~mm}$ of the atriovenous junctions of the pulmonary veins. This is done to reduce the risk of pulmonary vein stenosis, a recognised complication [37]. Hence a prior knowledge of these sites is very helpful. Furthermore, the likelihood of damage to the pulmonary vein branches is reduced by knowledge of ostial orientation and the distance to the first-order branch. The latter influences the size of the catheter selected.

A critical diagnosis to exclude is the presence of thrombus in the atrium or atrial appendage, which is an absolute contraindication to radiofrequency ablation [4]. Anatomical relationship to structures that can be damaged during ablation is also important. For example, there have been case reports of the left circumflex artery and oesophagus being injured [38, 39]. Finally, analysis of the extracardiac soft tissues and lungs is important to identify any important incidental findings.

CT can also be used to follow-up patients with complications of RFA, namely pulmonary vein stenosis (Fig. 14).

A summary of the important CT findings prior to RFA is summarised in Table 3. 


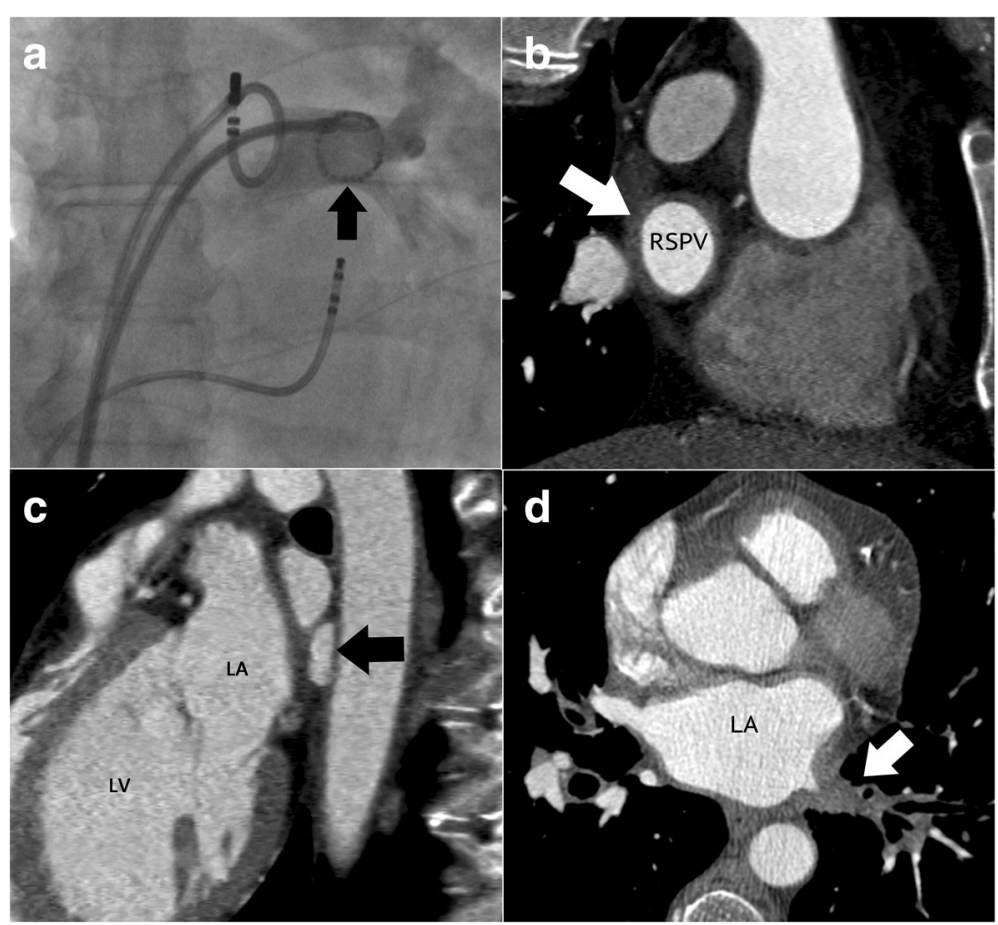

Fig. 14 Complications of radiofrequency ablation. a Anteroposterior fluoroscopic image of radiofrequency ablation (RFA). Radiofrequency coil (black arrow) is seen within the left inferior pulmonary vein. b Post-RFA. Axial maximum intensity projections (MIP) CT image showing soft tissue thickening around the ostium of the right superior pulmonary vein (arrow) post radiofrequency ablation, but no stenosis. c Sagittal CT image show narrowing of the left inferior pulmonary vein (LIPV) seen post ablation (arrow). $\mathbf{d}$ LIPV thrombosis post radiofrequency ablation. Axial CT image demonstrating occlusion of LIPV with thrombus

\section{Conclusion}

CT and MRI have refined assessment of pulmonary venous anatomy with variants and anomalies being increasingly recognized. APVD can be asymptomatic or non-specific in presentation and the presence of right heart dilatation should prompt particular scrutiny of the pulmonary venous anatomy. Pulmonary veins are a recognised source of ectopic foci triggering episodes of AF. Therefore mapping of the relevant anatomy is crucial, to facilitate clinical decisions and planning of surgical and catheter based interventions such as RFA. For these reasons, knowledge of anomalous pulmonary venous drainage and variant anatomy is important and the cardiac imaging specialist plays a

Table 3 Important findings to note on MDCT prior to RFA

- Identification of normal pulmonary vein anatomy

- Diameter of the ostia and their distance to the first-order branch

- Presence of supernumerary or conjoined veins, size of their ostia and distance to the first-order branches.

- Presence of anomalous pulmonary venous drainage

- Presence or absence of thrombus in the atrium or atrial appendage

- Incidental findings in the lungs or extra-cardiac soft tissues. crucial role, not only in these cases but also in the diagnostically challenging patient.

\section{Abbbreviations}

AF: Atrial fibrillation; APVD: Anomalous pulmonary venous drainage;

CT: Computed tomography; ECG: Electrocardiogram; IVC: Inferior vena cava; LA: Left atrium; LIPV: Left inferior pulmonary vein; LSPV: Left superior pulmonary vein; MRI: Magnetic resonance imaging; PAPVD: Partial anomalous pulmonary venous drainage; RA: Right atrium; RIPV: Right inferior pulmonary vein; RSPV: Right superior pulmonary vein; SVC: Superior vena cava; TAPVD: Total anomalous pulmonary venous drainage

\section{Acknowledgements}

Not applicable

\section{Financial support}

None

\section{Funding}

None

\section{Availability of data and materials}

Data sharing not applicable to this article as no datasets were generated or analysed during the current study.

\section{Authors' contributions}

SL contributed to manuscript drafting, revising the drafts, obtaining and formatting images. SW contributed to manuscript drafting, revising the drafts, creating Tables. EN contributed to manuscript drafting, revising the drafts, creating Tables. HM provided critical manuscript revisions and drafting. MY provided critical manuscript revisions and drafting. MH provided 
critical manuscript revisions and drafting. NM was responsible for review conception, provided database for image collection, and critical manuscript revisions. All authors read and approved the final manuscript.

\section{Ethics approval and consent to participate}

Not applicable

\section{Consent for publication}

Not applicable

\section{Competing interests}

The authors declare that they have no competing interests.

\section{Publisher's Note}

Springer Nature remains neutral with regard to jurisdictional claims in published maps and institutional affiliations.

\section{Author details}

1 Radiology Department, University Hospitals Bristol NHS Foundation Trust, Bristol Royal Infirmary, Upper Maudlin St, Bristol BS2 8HW, UK. ${ }^{2}$ Queen Elizabeth Hospital, Birmingham, UK.

Received: 1 January 2016 Accepted: 21 July 2017

\section{Published online: 26 August 2017}

\section{References}

1. Zylak CJ, Eyler WR, Spizarny DL, Stone CH. Developmental lung anomalies in the adult: radiologic-pathologic correlation. Radiographics. Radiological Society of North America; 2002;22 Spec No:S25-S43.

2. Latson LA, Prieto LR. Congenital and acquired pulmonary vein stenosis. Circulation. 2007;115(1):103-8.

3. Ghaye B, Szapiro D, Dacher JN, Rodriguez LM, Timmermans C, Devillers D, et al. Percutaneous ablation for atrial fibrillation: the role of cross-sectional imaging. Radiographics. 2003;23 Spec No:S19-S33; discussion S48-50.

4. Lacomis JM, Wigginton W, Fuhrman C, Schwartzman D, Armfield DR, Pealer KM. Multi-detector row CT of the left atrium and pulmonary veins before radio-frequency catheter ablation for atrial fibrillation. Radiographics. 2003; 23 Spec No:S35-S48; discussion S48-50.

5. Tsao H-M, Wu M-H, Yu W, Tai C-T, Lin Y-K, Hsieh M-H, et al. Role of right middle pulmonary vein in patients with paroxysmal Atrial fibrillation. J Cardiovasc Electrophysiol Blackwell Science Inc. 2001;12(12):1353-7.

6. Schwartzman D, Lacomis J, Wigginton WG. Characterization of left atrium and distal pulmonary vein morphology using multidimensional computed tomography. J Am Coll Cardiol. 2003;41(8):1349-57.

7. Jongbloed MRM, Dirksen MS, Bax JJ, Boersma E, Geleijns K, Lamb HJ, et al. Atrial fibrillation: multi-detector row CT of pulmonary vein anatomy prior to radiofrequency catheter ablation-initial experience. Radiology. 2005;234(3): 702-9.

8. Marom EM, Herndon JE, Kim YH, McAdams HP. Variations in pulmonary venous drainage to the left atrium: implications for radiofrequency ablation. Radiology. 2004:230(3):824-9.

9. Dillman JR, Yarram SG, Hernandez RJ. Imaging of pulmonary venous developmental anomalies. Am J Roentgenol. 2009;192(5):1272-85.

10. Demos TC, Posniak HV, Pierce KL, Olson MC, Muscato M. Venous anomalies of the thorax; 2004. p. 1139-50

11. Snellen H. A, van Ingen HC, Hoefsmit EC. Patterns of anomalous pulmonary venous drainage. Circulation. 1968;38(1):45-63.

12. Ammash NM, Seward JB, Warnes CA, Connolly HM, O'Leary PW, Danielson GK. Partial anomalous pulmonary venous connection: diagnosis by transesophageal echocardiography. J Am Coll Cardiol. 1997;29(6):1351-8.

13. van de Woestijne PC, Verberkmoes N, Bogers AJJC. Partial anomalous pulmonary venous connection (including scimitar syndrome). Multimed Man Cardiothorac Surg MMCTS / Eur Assoc Cardio-Thoracic Surg. 2013; 2013(February):mmt001.

14. Porres DV, Morenza OP, Pallisa E, Roque A, Andreu J, Martínez M. Learning from the pulmonary veins. Radiographics. 2013;33(4):999-1022.

15. Kivistö $\mathrm{S}$, Hänninen $H$, Holmström M. Partial anomalous pulmonary venous return and atrial septal defect in adult patients detected with 128-slice multidetector computed tomography. J Cardiothorac Surg BioMed Central Ltd. 2011;6(1):126.
16. Dyme JL, Prakash A, Printz BF, Kaur A, Parness IA, Nielsen JC. Physiology of isolated anomalous pulmonary venous connection of a single pulmonary vein as determined by cardiac magnetic resonance imaging. Am J Cardiol. 2006:98(1):107-10.

17. Pauwaa S, Farzaneh-Far A. Isolated partial anomalous pulmonary venous return with intact atrial septum: a rare but treatable cause of pulmonary hypertension in adults. Eur Hear J Cardiovasc Imaging. 2014;15(7):830.

18. Berrocal T, Madrid C, Novo S, Gutiérrez J, Arjonilla A, Gómez-León N. Congenital anomalies of the tracheobronchial tree, lung, and mediastinum: embryology, radiology, and pathology. Radiographics. 2009;24(1):e17.

19. Chowdhury MM, Chakraborty S. Imaging of congenital lung malformations. Semin Pediatr Surg Elsevier. 2015;24(4):168-75

20. Bo I, Carvalho JS, Cheasty E, Rubens M, Rigby ML. Variants of the scimitar syndrome. Cardiol Young. 2016;26(5):941-7.

21. Legras A, Guinet C, Alifano M, Lepilliez A, Regnard JF. A case of variant scimitar syndrome. Chest. 2012;142(4):1039-41.

22. Javangula K, Cole J, Cross M, Kay PH. An unusual manifestation of left partial anomalous pulmonary venous connection. Interact Cardiovasc Thorac Surg. 2010;11(6):846-7.

23. Dillon EH, Camputaro C. Partial anomalous pulmonary venous drainage of the left upper lobe vs duplication of the superior vena cava: distinction based on CT findings. Am J Roentgenol. 1993:160(2):375-9.

24. Kokotsakis J, Anagnostakou V, Almpanis G, Paralikas I, Nenekidis I, Kratimenos T, et al. Cor triatriatum presenting as heart failure with reduced ejection fraction: a case report. J Cardiothorac Surg. 2011;6:83.

25. Ferguson EC, Krishnamurthy R, Oldham S. A a. Classic imaging signs of congenital cardiovascular abnormalities. Radiographics. 2007;27(5):1323-34.

26. Vyas HV, Greenberg SB, Krishnamurthy R. MR imaging and CT evaluation of congenital pulmonary vein abnormalities in neonates and infants. Radiographics. 2012;32(1):87-98.

27. Seale AN, Uemura H, Webber SA, Partridge J, Roughton M, Ho SY, et al. Total anomalous pulmonary venous connection: morphology and outcome from an international population-based study. Circulation. 2010;122(25): 2718-26.

28. Boyd K, Clark D, Colthart A, Donald P, Forbes C, Fox K, et al. Consensus conference on atrial fibrillation in hospital and general practice. Scott Med J. 1998:43(6):166-7.

29. Wolf PA, Abbott RD, Kannel WB. Atrial fibrillation as an independent risk factor for stroke: the Framingham study. Stroke. 1991;22(8):983-8.

30. Pelosi FJ, Morady F. Evaluation and management of atrial fibrillation. Med Clin North Am. 2001;85(2):225-44. ix

31. Shah DC, Haïssaguerre M, Jaïs $P$, Hocini M, Yamane T, Deisenhofer I, et al. Electrophysiologically guided ablation of the pulmonary veins for the curative treatment of atrial fibrillation. Ann Med. 2000;32(6):408-16.

32. Ho SY, Sanchez-quintana D, Cabrera JA, Anderson RH. Anatomy of the left atrium: implications for radiofrequency ablation of Atrial fibrillation. J Cardiovasc Electrophysiol. 1999;10:1525-33.

33. Ho SY, Cabrera JA, Tran VH, Farré J, Anderson RH, Sánchez-Quintana D. Architecture of the pulmonary veins: relevance to radiofrequency ablation. Heart. 2001:86(3):265-70.

34. Haissaguerre M, Jais P, Shah DC, Takahashi A, Hocini M, Quiniou G, et al. Spontaneous initiation of Atrial fibrillation by ectopic beats originating in the pulmonary veins. N Engl J Med. 1998;339(10):659-66.

35. Niinuma H, George RT, Arbab-Zadeh A, Lima JA, Henrikson CA. Imaging of pulmonary veins during catheter ablation for atrial fibrillation: the role of multi-slice computed tomography. Europace. 2008;10(Suppl 3):iii14-21.

36. Cronin P, Sneider MB, Kazerooni EA, Kelly AM, Scharf C, Oral H, et al. MDCT of the left atrium and pulmonary veins in planning radiofrequency ablation for atrial fibrillation: a how-to guide. AJR Am J Roentgenol. 2004;183(3):767-78.

37. Shroff GS, Guirguis MS, Ferguson EC, Oldham SAA, Kantharia BK. CT imaging of complications of catheter ablation for atrial fibrillation. Clin Radiol. 2014; 69(1):96-102.

38. Takahashi $Y$, Jaïs $P$, Hocini $M$, Sanders $P$, Rotter $M$, Rostock $T$, et al. Acute occlusion of the left circumflex coronary artery during mitral isthmus linear ablation. J Cardiovasc Electrophysiol. 2005;16(10):1104-7.

39. Siegel MO, Parenti DM, Simon GL. Atrial-esophageal fistula after Atrial radiofrequency catheter ablation. Clin Infect Dis. 2010;51(1):73-6. 